\title{
Rapid Synaptic Remodeling in the Adult Somatosensory Cortex following Peripheral Nerve Injury and Its Association with Neuropathic Pain
}

\author{
Sun Kwang Kim ${ }^{1,2}$ and Junichi Nabekura ${ }^{1,3,4}$ \\ ${ }^{1}$ Division of Homeostatic Development, National Institute for Physiological Sciences, Okazaki 444-8585, Japan, ${ }^{2}$ Acupuncture \& Meridian Science Research Center, \\ Kyung Hee University, Seoul 130-701, Republic of Korea, ${ }^{3}$ Core Research for Evolutional Science and Technology, Japan Science and Technology Agency, Saitama \\ 332-0012, Japan, and ${ }^{4}$ Department of Physiological Sciences, The Graduate School for Advanced Study, Hayama 240-0193, Japan
}

Structural and functional plastic changes in the primary somatosensory cortex (S1) have been observed following peripheral nerve injury that often leads to neuropathic pain, which is characterized by tactile allodynia. However, remodeling of cortical connections following injury has been believed to take months or years; this is not temporally correlated with the rapid development of allodynia and S1 hyperexcitability. Here we first report, by using long-term two-photon imaging of postsynaptic dendritic spines in living adult mice, that synaptic connections in the $S 1$ are rewired within days following sciatic nerve ligation through phase-specific and size-dependent spine survival/growth. Spine turnover in the $S 1$ area corresponding to the injured paw markedly increased during an early phase of neuropathic pain and was restored in a late phase of neuropathic pain, which was prevented by immediate local blockade of the injured nerve throughout the early phase. New spines that generated before nerve injury showed volume decrease after injury, whereas more new spines that formed in the early phase of neuropathic pain became persistent and substantially increased their volume during the late phase. Further, preexisting stable spines survived less following injury than controls, and such lost persistent spines were smaller in size than the surviving ones, which displayed long-term potentiation-like enlargement over weeks. These results suggest that peripheral nerve injury induces rapid and selective remodeling of cortical synapses, which is associated with neuropathic pain development, probably underlying, at least partially, long-lasting sensory changes in neuropathic subjects.

\section{Introduction}

Peripheral nerve injury triggers maladaptive plastic changes along the somatosensory system so that altered nociceptive signal processing, represented by tactile allodynia (painful response to innocuous mechanical stimuli), occurs (Costigan et al., 2009). There is a general consensus that injury-induced early afferent barrage can drive subsequent alterations in the CNS, including the cortex, that in turn contribute to neuropathic pain (Melzack et al., 2001; Devor, 2006). In functional brain imaging studies, nerve-injured patients and animals show enhanced excitation, somatotopic reorganization, and changed cortical thickness in

\footnotetext{
Received Jan. 19, 2011; revised Feb. 17, 2011; accepted Feb. 18, 2011

Author contributions:S.K.K. and J.N. designed research;S.K.K. performed research;S.K.K. and J.N. analyzed data; S.K.K. and J.N. wrote the paper.

This work was supported by the Core Research for Evolutional Science and Technology grant from the Japan Science and Technology Agency and Grant-in-Aid for Scientific Research (A) (22240042) from the Japan Society for the Promotion of Science (JSPS) to J.N.; and the JSPS foreign researcher fellowship (P08456), Grant-in-Aid for Scientific Research (20-08456) from JSPS, and Basic Science Research Program through the National Research Foundation funded by the Ministry of Education, Science, and Technology, Korea (R11-2005-014) to S.K.K. We thank H. Ishibashi, T. Nemoto, H. Wake, Y. Takatsuru, K. Eto, H. Inada, and C. Yin for excellent technical advice and critical discussions on the experiments and manuscript; M. Kano for the generous gift of Elvax beads; M. Yoshitomo and T. Ohba for expert technical assistance; and S. Park for providing a mouse illustration.

The authors declare no competing financial interests.

Correspondence should be addressed to Dr. Junichi Nabekura, Division of Homeostatic Development, National Institute for Physiological Sciences, 0kazaki 444-8585, Japan. E-mail: nabekura@nips.ac.jp.

DOI:10.1523/JNEUROSCI.0328-11.2011

Copyright $\odot 2011$ the authors $\quad 0270-6474 / 11 / 315477-06 \$ 15.00 / 0$
}

the $\mathrm{S} 1$, the amount of which is highly correlated with the degree of allodynia (Peyron et al., 2004; DaSilva et al., 2008; Cha et al., 2009). However, it remains unknown how and to what extent neuronal connections in the $\mathrm{S} 1$ are remodeled at the individualsynapse level following peripheral nerve injury and how such remodeling is associated with neuropathic pain.

Interestingly, learning and memory and neuropathic pain share similar mechanisms, as exemplified by "long-term potentiation (LTP)" and "central sensitization," respectively, both of which are use-dependent synaptic plasticity (Ji et al., 2003; Zhuo, 2008). Recent advances in long-term in vivo imaging have revealed that novel sensory experiences induce significant rewiring of cortical circuits, probably underlying long-term memory storage, which is achieved rapidly by changes in formation and elimination of dendritic spines that receive most excitatory synaptic inputs (Holtmaat et al., 2006; Hofer et al., 2009; Yang et al., 2009).

Here, we used long-term two-photon imaging to longitudinally trace the dynamics of individual spines in the S1 of living mice with neuropathic pain induced by the partial sciatic nerve ligation (PSL), a well characterized mouse model (Malmberg and Basbaum, 1998). We report that rewiring of cortical connections following PSL occurs within days, modifying the current belief that it takes much longer periods (Florence et al., 1998; Zhuo, 2008). We also found phase-specific and size-dependent growth/ survival of newly formed spines and previously persistent spines, respectively, which might provide a structural basis of how local 
cortical circuits change their response property to sensory information during neuropathic pain development.

\section{Materials and Methods}

All animal experiments were approved by the Animal Research Committee of the National Institutes of Natural Sciences. Every effort was made to minimize the number and suffering of animals.

PSL, tactile allodynia, and somatosensory-evoked potentials. Under isoflurane anesthesia $(\sim 2.0 \%)$, the right sciatic nerve of male C57BL/6 mice (3 months old) was exposed at high-thigh level and one-third to one-half the diameter of the nerve was ligated with 9-0 suture. For sham operation, the nerve was exposed, but left intact. For assessing tactile allodynia, the mice were placed in a transparent box with mesh floor and eight calibrated von Frey hairs (0.07-4.0 g bending force) were pressed perpendicularly against the plantar surface of the hindpaw until slight buckling. The $50 \%$ paw withdrawal threshold was determined by the Dixon's up-down method. Cortical field potentials evoked by low-intensity electrical stimulation $(3 \mathrm{~V}, 0.5 \mathrm{~ms})$ of the right hindpaw were measured in the layer 1 of the left S1 as described previously (Takatsuru et al., 2009).

Long-term in vivo imaging. Male C57BL/6 mice (2 months old) expressing GFP in a small subset of pyramidal neurons (M-line) (Feng et al., 2000) were anesthetized with ketamine $(0.13 \mathrm{mg} / \mathrm{g})$ and xylazine $(0.01$ $\mathrm{mg} / \mathrm{g}$, i.p.). After the scalp incision, a small circular craniotomy $(2-3 \mathrm{~mm}$ in diameter) was performed on the skull above the left $\mathrm{S} 1$ hindlimb area ( $0.5 \mathrm{~mm}$ posterior, $1.5 \mathrm{~mm}$ lateral to the bregma), leaving intact the dura, which was covered with a glass coverslip (Holtmaat et al., 2005). Repeated imaging experiments ( 3 dintervals) were started 1 month after the cranial window implantation, because general mechanical sensitivity increased for 2 weeks after craniotomy and returned to normal at 4 weeks (our unpublished observation). Although it was suggested that the openskull preparation is associated with high spine turnover during the first 4-6 weeks after craniotomy (Xu et al., 2007), we found no turnover difference between 5 and 8 weeks after craniotomy $(13.6 \pm 1.2 \%, n=7$ and $13.0 \pm 2.4 \%, n=3$ mice, respectively). Minimal isoflurane anesthesia $(\sim 1.5 \%)$ was used every imaging session for $30-40 \mathrm{~min}$, which did not affect general mechanical sensitivity. On the first imaging session, the S1 hindpaw area was determined using intrinsic optical signal imaging (Hofer et al., 2009).

A Ti:sapphire laser (Mai Tai HP, Spectra-Physics) was tuned to the excitation wavelength for GFP (950 nm) (Wake et al., 2009). Only 1-2 neurons were usually identified within the $S 1$ hindpaw area and a centered cell was selected when $\geq 2$ cells existed. Low-magnification image stacks $(512 \times 512$ pixels, $0.41 \mu \mathrm{m} /$ pixel, $250-400$ optical planes, $2-2.5$ $\mu \mathrm{m} z$-step) of the identified cell were collected on the second imaging session using a FV1000MPE laser scanning microscope and a waterimmersion objective lens (60×, NA 0.9, Olympus) and matched to the blood vessel pattern on the brain surface to relocate the imaging regions. On the third imaging session, 2-4 segments of the distal tuft dendrites, containing second- and higher-order branches, within 100 $\mu \mathrm{m}$ below the brain surface (layer 1 ) were randomly chosen for further spine imaging by the experimenter, who was unaware whether the animals would subsequently receive PSL or other treatments. Since then, high-magnification $(0.08 \mu \mathrm{m} /$ pixel, $15-30$ optical planes, $0.5 \mu \mathrm{m} z$-step) imaging of the selected dendritic segments was performed every $3 \mathrm{~d}$ for $2-4$ weeks.

Elvax-TTX application. Elvax (non-inflammatory drug delivery system) containing tetrodotoxin (TTX, final concentration $200 \mu \mathrm{M}$ ) or physiological saline was prepared as described previously (Kakizawa et al., 2005). A small piece of Elvax $\left(2 \times 4 \mathrm{~mm}^{2}\right)$ of which estimated working duration is $6 \mathrm{~d}$ was placed just proximal to the injured site of the sciatic nerve. Elvax implantation alone (i.e., Elvax-saline) did not affect general mechanical sensitivity in normal mice and allodynic behavior in PSL mice (see Fig. 2G).

Image analysis. MetaMorph (Molecular Devices) and ImageJ (http://rsbweb.nih.gov/ij/) were used to analyze individual spines on the same dendrites from three-dimensional image stacks, blind to the experimental conditions. Detailed criteria for scoring the spines have been described previously (Holtmaat et al., 2005). We included all types of dendritic protrusions in analysis, but not the spines on dendritic branch tips, which slightly change their morphology during the long imaging period. Spine gain and loss rates were determined as the percentages of spine formation and elimination between two successive imaging sessions, relative to the total spine number in the former session. Spine turnover was defined as the number of gained and lost spines divided by twice the total spine number in the former session. We defined the spines that had survived for at least three consecutive imaging sessions (6-8d) as "stable" or "persistent" (see Figs. $3 A, 4 A$ ), because they might all have synapses (Knott et al., 2006) and are likely to survive for weeks (Holtmaat et al., 2005; Yang et al., 2009). Only spines that clearly protruded from parent dendrites were included in the analysis of integrated spine brightness, which was calculated in the best focal section by summing the intensity of all pixels comprising a spine head after background subtraction, divided by mean intensity of the adjacent dendritic shaft.

Statistics. Data are presented as mean \pm SEM. $p$ values were calculated using Mann-Whitney test for independent samples and repeatedmeasures ANOVA followed by Wilcoxon signed-rank test for paired comparisons of longitudinal measurements.

\section{Results}

PSL injury, but not sham operation, markedly increased mechanical sensitivity of the injured paw, with the increase being detected within the first day, peaking on day 6 , and persisting for at least 1 month (Fig. $1 A$ ), differentiating in our experimental condition between the early ( $\leq 6 \mathrm{~d}$ ) and late ( $\geq 6 \mathrm{~d}$ ) phases of neuropathic pain (Kawasaki et al., 2008). Somatosensory-evoked potentials significantly increased in the early phase and to a greater extent in the late phase $(p<0.04)$ (Fig. $1 B$ ), indicating that the S1 excitability increases phase dependently. From these time-matched behavioral and electrophysiological results, together with experience/activity-dependent spine plasticity (Bhatt et al., 2009), we hypothesized that spine turnover in the S1 of neuropathic mice would be enhanced in a phase-dependent manner. To explore this, we repeatedly imaged the same apical tuft of layer 5 pyramidal cells in the functionally defined S1 areas of M-line mice through a chronic cranial window by using twophoton microscopy (Fig. $1 C-E$ ). Layer 5 pyramidal neurons are the major output cells in the S1, and their distal tuft dendrites encode information about somatosensory stimuli (Murayama et al., 2009).

High-resolution imaging revealed unexpected results, showing a marked increase $(\sim 58 \%)$ in spine turnover during the early phase, but restoration in the late phase when compared with preinjury or time-matched controls (PSL $+3 \mathrm{~d}: p<0.02$; PSL + $6 \mathrm{~d}: p<0.003$; other periods: $p>0.07$ ) (Fig. $2 A$ ). Such turnover change is injury- and region-specific, because little change was found in the S1 hindpaw area of sham-operated mice $(p>0.8)$ (Fig. $2 A$ ) and in the barrel cortex of PSL mice (turnover rate: $10.1 \pm 2.0 \%$ at baseline, $10.0 \pm 1.1 \%$ at PSL $+3 \mathrm{~d}$, and $9.5 \pm$ $2.9 \%$ at PSL $+6 \mathrm{~d} ; n=145$ spines from 6 dendrites of 2 mice; $p>$ $0.9)$. In control groups, spine gain and loss rates were not changed throughout the experimental period and similar to each other $(p>0.1)$ (Fig. $2 B, C)$, resulting in little change in turnover $(p>$ 0.5 ) (Fig. $2 A$ ) and density ( $p>0.1$ ) (Fig. $2 D$ ). Conversely, spine gain rate in PSL mice showed a striking increase $(\sim 68 \%)$ during the early phase $(\leq \mathrm{PSL}+6 \mathrm{~d} ; p<0.03)$ and returned to baseline in the late phase ( $\geq \mathrm{PSL}+9 \mathrm{~d} ; p>0.9)$ (Fig. $2 B$ ). Spine loss rate also increased following injury, but to a lesser degree $(\sim 44 \%)$ for a more prolonged period ( $\leq$ PSL $+9 \mathrm{~d} ; p<0.05$ ) (Fig. $2 C$ ). Consequently, spine density had significantly increased at the end of the early phase $(p<0.04)$ and decreased to control level during the subsequent $6 \mathrm{~d}(p>0.6)$ (Fig. $2 D)$. Since the majority of new spines were transient $(\sim 80 \%)$ (Fig. $3 C$ ), our data suggest that new cortical connections are rapidly generated after neuro- 
A

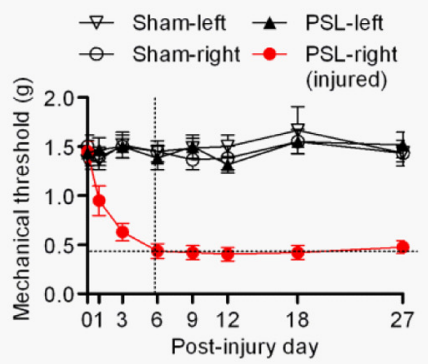

C

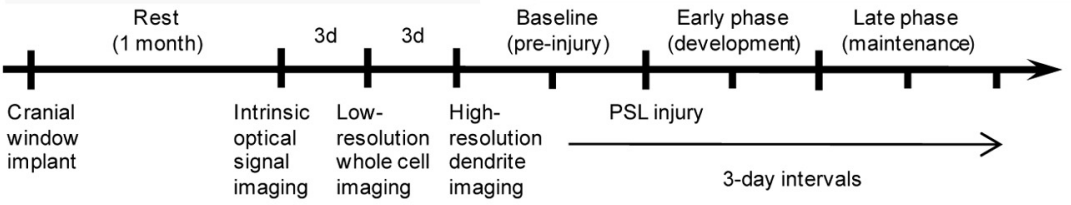

D
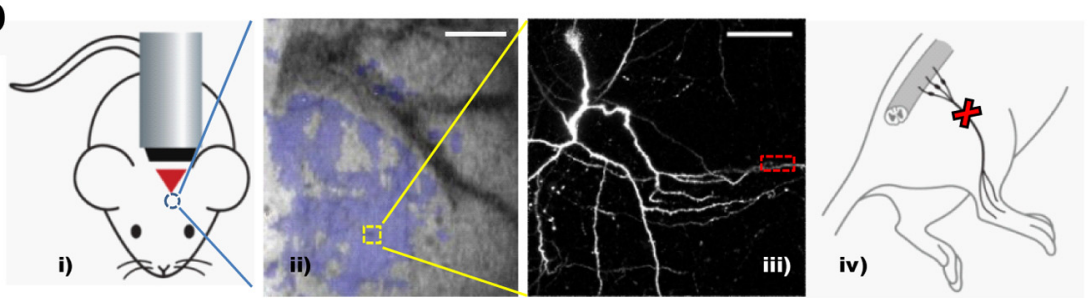

$\mathbf{E}$

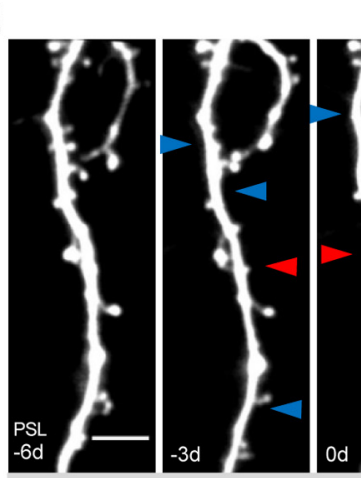

Baseline (pre-injury) phase

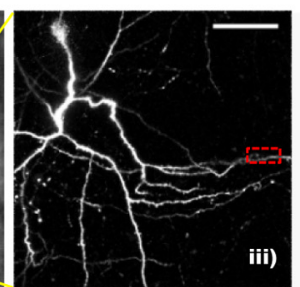

B

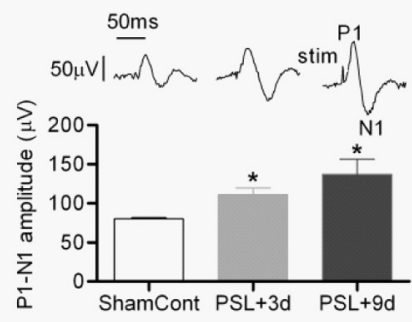

Injury

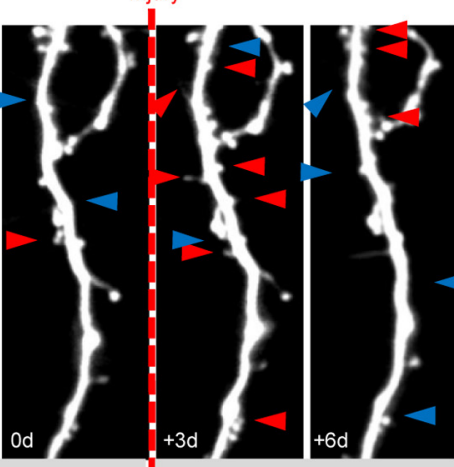

Early (development) phase

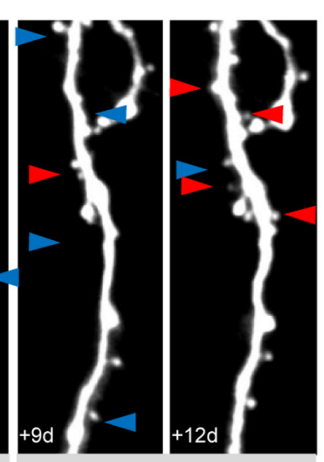

Late (maintenance) phase

Figure 1. Tactile allodynia, S1 excitability, and long-term imaging. $\boldsymbol{A}$, Development and maintenance of tactile allodynia following PSL ( $n=12$, pooled of 6 wild-type and 6 M-line mice) as shown by reduced mechanical threshold. Sham, Shamoperated mice $(n=12)$. Left and right, Left and right hindpaws. $\boldsymbol{B}$, Top, Representative traces of somatosensory-evoked potentials in the S1 showing the typical primary response, positive-negative (P1-N1) wave. Bottom, Amplitude of P1-N1 wave increased in the early phase (PSL $+3 d, n=3)$ and to a greater extent in the late phase (PSL $+9 d, n=3)\left({ }^{*} p<0.04\right.$ vs ShamCont: pooled of 3 sham-operated and 2 normal control mice). C, Timeline of long-term imaging experiments. Di, Schematic of in vivo imaging of the S1 (blue circle); Dii, intrinsic optical signal map of the S1 hindpaw area (scale bar, $500 \mu \mathrm{m}$ ); Diii, low-magnification z-projection image of a layer 5 pyramidal cell (scale bar, $50 \mu \mathrm{m}$ ) taken in the region indicated in Dii (yellow square); Div, schematic of PSL injury. $\boldsymbol{E}$, High-magnification repeated imaging of the same dendritic segment shown in Diii (red box). All types of dendritic protrusions are included in analysis, except the spines near dendritic tips. Arrowheads indicate spines generated (red) or eliminated (blue) when compared with the previous imaging session. Scale bar, $5 \mu \mathrm{m}$.

pathic injury with simultaneous, but relatively slow, elimination of preexisting circuits, followed by refinement of the new connections. Because early afferent hyperactivity is the key driver for development of CNS changes and allodynia (Devor, 2006), we conducted local blockade of the injured nerve throughout the early phase, which prevents afferent ectopic firing even after its action is finished (Xie et al., 2005), by directly implanting an Elvax-TTX immediately after PSL. Such temporary blockade prevented the increase of spine gain and loss in the $S 1(p>0.3)$ (Fig. $2 E, F)$ and completely inhibited allodynia (Fig. $2 G$ ).

We next quantified new spines and tracked their long-term fates. The density of new persistent (NP) spines that appeared in

the early phase (postinjury NP spines) was significantly higher than that of NP spines that appeared before PSL (preinjury NP spines) and in controls $(p<0.04)$ (Fig. $3 B)$. However, there was no difference in the ratio of NP spines to total new spines between groups $(p>0.1)$ (Fig. $3 C$ ), as suggested in the visual cortex during monocular deprivation (Hofer et al., 2009). Since synaptic strength is positively correlated with spine size (Kasai et al., 2003), we followed the volume of individual NP spines by measuring integrated spine brightness, which is monotonically related to spine volume (Holtmaat and Svoboda, 2009). NP spines in control groups showed gradual volume increase following their appearance (Fig. $3 D, E$ ). In the PSL group, however, the volume of preinjury NP spines decreased during the early phase (PSL $+6 \mathrm{~d}: p<0.02$ ) (Fig. $3 D$ ), whereas postinjury NP spines substantially increased their size during the late phase (PSL $+9 \mathrm{~d}: p<0.05)$ (Fig. $3 E$ ), indicating the phase-specific strengthening and weakening of synaptic weight of new circuits.

To understand how neuropathic injury affects previously persistent circuits, we compared the survival of preexisting stable spines (Fig. 4A) and their estimated lifetime between PSL and control groups. Before nerve injury, there was no difference between groups in the survival rate of initial spines observed at the first highmagnification imaging session (PSL: $84.5 \pm 1.6 \%$ at $-3 \mathrm{~d}$ and $77.3 \pm 1.9 \%$ at $0 \mathrm{~d}$; ShamCont: $85.9 \pm 2.1 \%$ at $-3 \mathrm{~d}$ and $78.5 \pm 2.7 \%$ at $0 \mathrm{~d} ; p>0.5$ ), indicating that the proportion of preexisting stable spines at day 0 was similar. However, the survival difference in preexisting stable spines emerged after injury, and only $69.2 \%$ of them survived over the experimental period in PSL mice, while $81.5 \%$ did in controls (PSL $+12 \mathrm{~d}: p<0.03$ ) (Fig. $4 B$ ). Simple estimation based on the above data further suggests that $69.1 \%$ of preexisting stable spines in controls might persist for life, but only $31.8 \%$ in PSL mice could (Fig. 4B). We additionally tracked the size of lost persistent (LP) and always-present (AP) spines (Fig. 4A). LP spines in PSL mice had been significantly smaller in size before injury (day 0$)$ than AP spines $(p<0.02)$ (Fig. $4 C$ ), although the distribution was varied in AP spines. Similar results were observed in control groups $(p<0.002)$, and there was no difference in either LP or AP spine size between PSL and control groups $(p>0.5)$ (Fig. $4 C$ ). Intriguingly, normalized brightness of AP spines significantly increased over the first $3 \mathrm{~d}$ following PSL $(p<0.03)$ and only partially decreased over the subsequent weeks, displaying LTP-like spine volume increase (Matsuzaki et al., 2004) on average (Fig. 4D). Such increase in AP spine brightness at PSL $+3 \mathrm{~d}$ was inhibited by immediate local nerve block- 

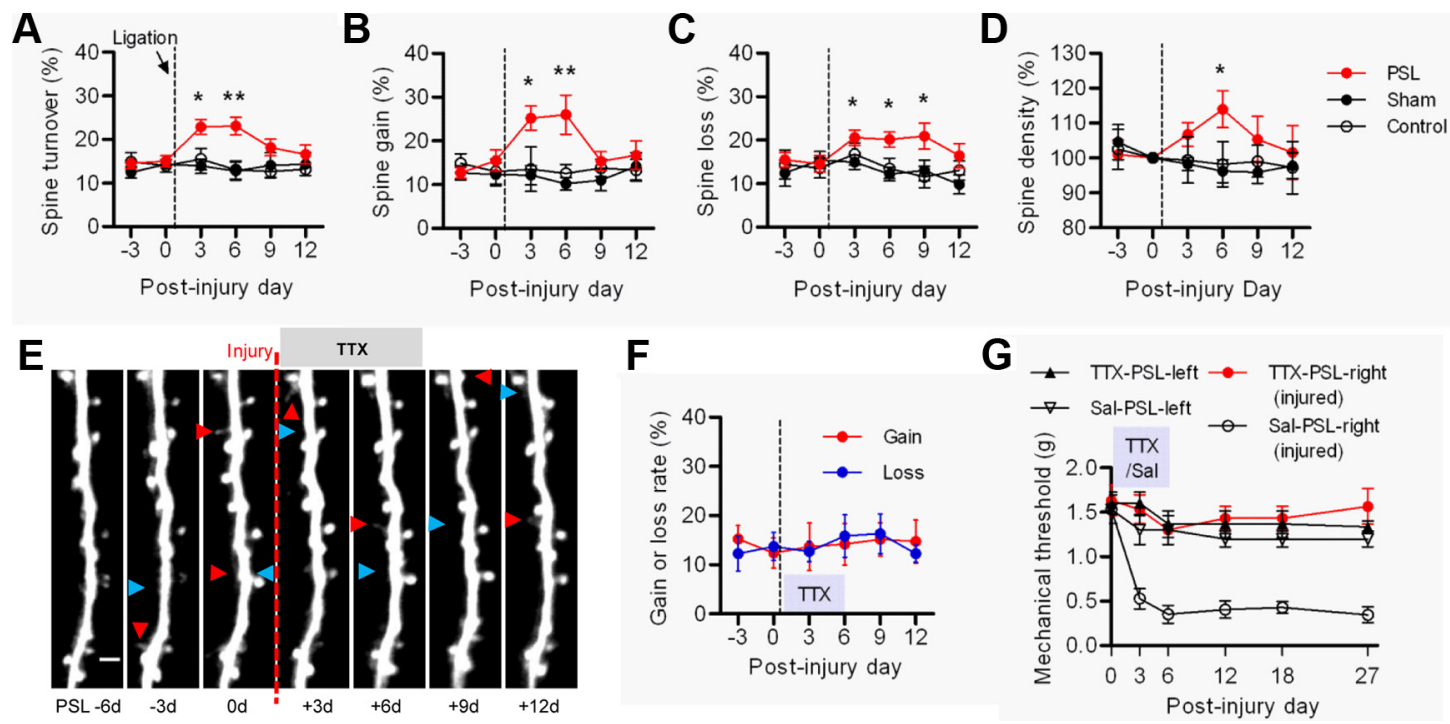

Figure 2. Change in spine dynamics following PSL injury and its prevention by immediate local nerve blockade. $\boldsymbol{A}$, Spine turnover rate $\left({ }^{*} p<0.02,{ }^{* *} p<0.003\right) . \boldsymbol{B}$, Spine gain rate $\left({ }^{*} p<0.03\right.$, $\left.{ }^{* *} p<0.007\right)$. C, Spine loss rate ( $\left.{ }^{*} p<0.05\right)$. D, Normalized spine density ( $\left.{ }^{*} p<0.04\right)$. Control, 356 spines from 9 dendrites in 3 mice; Sham, 386 spines from 11 dendrites in 4 mice; PSL, 522 spines from 12 dendrites in 5 mice. $\boldsymbol{E}$, Representative images of the same dendrite taken before and after PSL with local nerve blockade using Elvax-TTX implantation. Arrowheads indicate spines generated (red) or eliminated (blue) when compared with the previous session. Scale bar, $2 \mu \mathrm{m}$. $\boldsymbol{F}, \mathbf{G}$, Local nerve blockade throughout the early phase of neuropathic pain inhibited the increase of spine gain and loss ( 255 spines from 6 dendrites in 3 mice, $\boldsymbol{F}$ ) and development of allodynia (each group: $n=6, \boldsymbol{G}$ ).

A

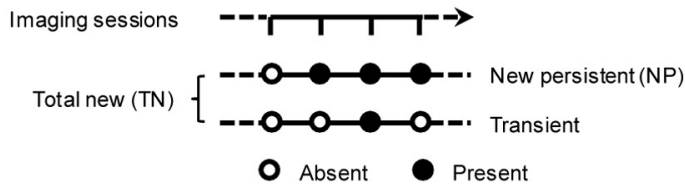

B

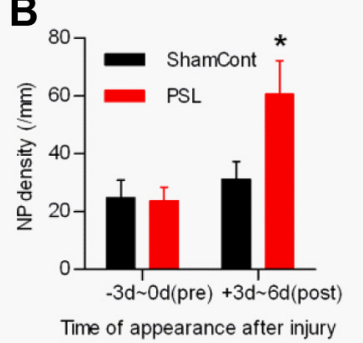

C

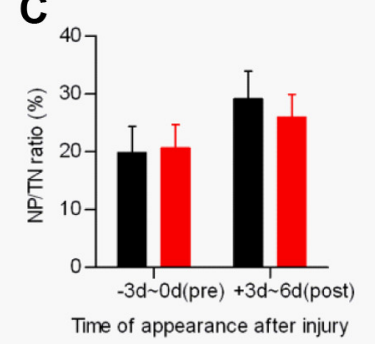

E
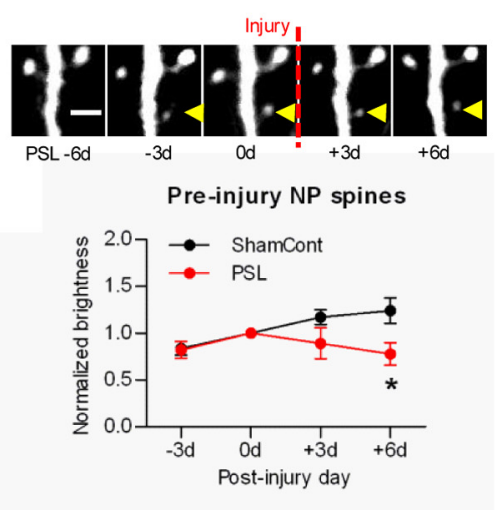

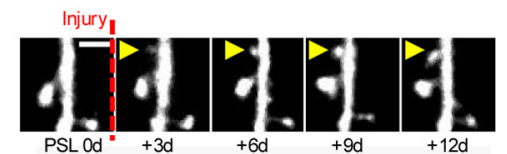

Post-injury NP spines

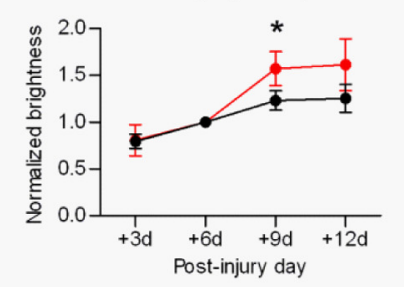

Figure 3. Phase-specific survival/growth of new spines. $\boldsymbol{A}$, Schematic of transient, NP, and total new (TN) spines. $\boldsymbol{B}$, Density of preinjury and postinjury NP spines $\left({ }^{*} p<0.04\right)$. $\boldsymbol{C}$, Ratio of NP spines to TN spines. ShamCont, 17 dendrites from 7 mice; PSL, 12 dendrites from 5 mice. $D$, Preinjury NP spines $(n=9)$ increase in normalized brightness before PSL, but gradually decrease after injury $\left[{ }^{*} p<0.02\right.$ vs ShamCont $\left.(n=14)\right]$. , Postinjury NP spines $(n=10)$ show a marked increase in brightness during the late phase of neuropathic pain $\left[{ }^{*} p<0.05\right.$ vs ShamCont $\left.(n=15)\right]$. Yellow arrowheads indicate NP spines. Scale bars, $2 \mu \mathrm{m}$.

ade (Fig. 4D). Further analysis revealed that individual AP spines showing long-lasting enlargement throughout the experimental period (Fig. $4 E$ ) are widely dispersed in size (Fig. $4 C$, red circles), even though the degree of increase in normalized brightness tends to be higher in small AP spines than in large ones (Fig. $4 E$ ).

\section{Discussion}

This study, to our knowledge, is the first to investigate long-term structural dynamics of individual neuronal circuits in the intact cortex following peripheral nerve injury and its association with chronic pain. Considering the time course of allodynia and S1 hyperexcitability (Fig. $1 A, B$ ) together, our data on spine dynamics (Fig. 2) suggest that nerve injury-specific remodeling of cortical circuits occurs mainly within the early phase of neuropathic pain, which is surprisingly fast, because it has been believed to take months or years (Florence et al., 1998; Zhuo, 2008).

Long-term in vivo imaging studies have focused on changes in spine turnover during sensory manipulation and suggested the increase of new persistent spines by such manipulation as longterm memory traces (Bhatt et al., 2009; Holtmaat and Svoboda, 2009). However, it remains unsolved whether new spines generated before manipulation as a baseline process grow normally when they encounter sensory change and how new spines formed after manipulation grow during prolonged sensory alteration. We demonstrate that the size of preinjury NP spines increases after appearance, but decreases following PSL, whereas postinjury NP spines markedly increased their volume during the late phase of neuropathic pain. These data suggest that preinjury NP 
A
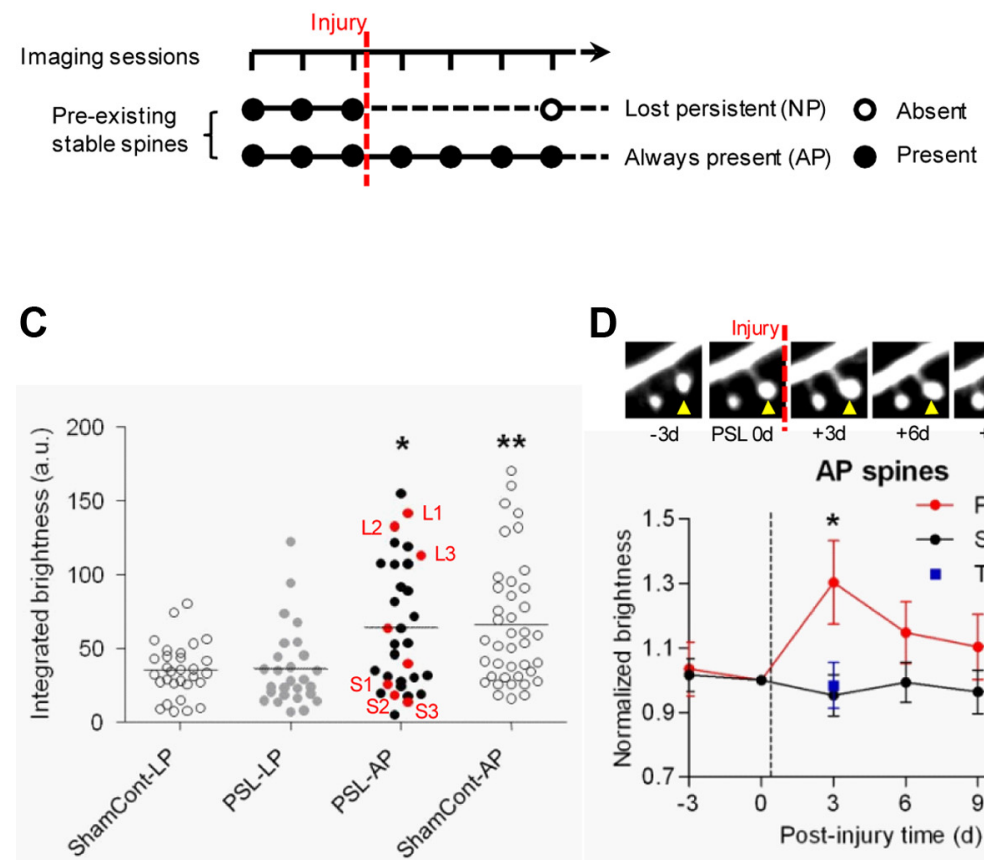

D
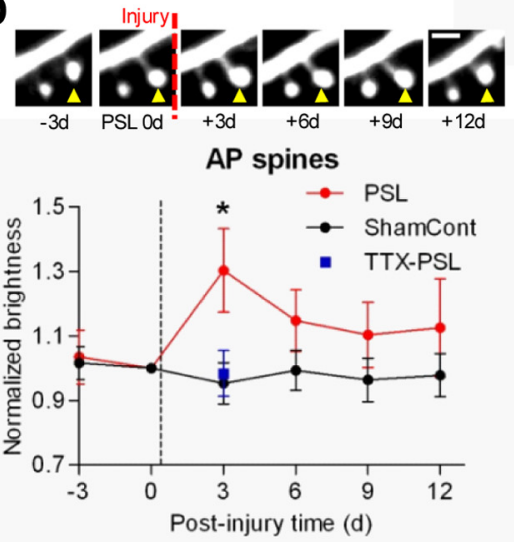

B
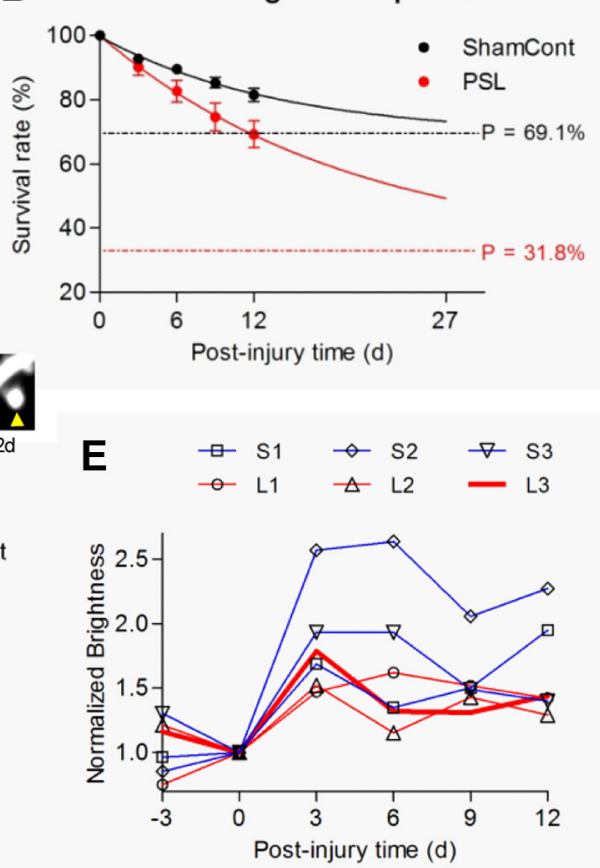

Figure 4. Size-dependent elimination of preexisting stable spines and LTP-like long-lasting enlargement of always-present spines following neuropathic injury. $A$, Schematic of LP, AP, and preexisting stable spines. $\boldsymbol{B}$, Survival rate of preexisting stable spines fitted with one exponential $\left(S=T e^{-d / \tau}+P\right.$, where $S$ is the survival rate, $T$ is an estimate of the percentage of spines that will ultimately disappear with a time constant $\tau$, and $P$ is an estimate of the percentage of spines that persist throughout life). C, Comparison of integrated brightness of AP (PSL: $n=32 ;$ ShamCont: $n=$ 40) and LP spines (PSL: $n=28$; ShamCont: $n=30$ ) just before injury (day $0 ;{ }^{*} p<0.02,{ }^{* *} p<0.002$ ). Red circles $(n=8)$ indicate individual AP spines showing long-lasting enlargement (criteria: $>30 \%$ increase in brightness at PSL $+3 \mathrm{~d}$ and $>10 \%$ increase at all subsequent time points, based on the graph in $\mathbf{D})$. D. Top, Representative images of AP spines before and after PSL. Scale bars, $2 \mu \mathrm{m}$. Note that a large AP spine (yellow arrowhead, corresponding to " $L 3$ " in $C, E$ ) shows long-lasting enlargement over weeks following injury. Bottom, LTP-like increase in normalized brightness of AP spines ( $n=32$ ) after PSL injury [ ${ }^{*} p<0.03$, vs ShamCont $\left.(n=40)\right]$. Note that Elvax-TTX implantation (blue square) immediately after injury blocked the increase in AP spine brightness at PSL + 3 d. $\boldsymbol{E}$, Long-term traces of normalized brightness in three large (L1-L3) and small (S1-S3) AP spines indicated in C.

spines, formed for the purpose of ordinary somatosensory functions, would be weakened in synaptic strength by reduced synaptic input through altered sensory signaling following PSL, whereas postinjury NP spines might be generated to handle abnormal neuropathic signals and increase their synaptic weight by amplified inputs. Alternatively, volume decrease of preinjury NP spines might reflect the adaptive process to generally increased activity (homeostatic plasticity) (Pozo and Goda, 2010).

Previous in vitro studies have demonstrated that spine head size reflects spine age as well as synaptic strength (Kasai et al., 2003; Yasumatsu et al., 2008), which is supported by in vivo data in control conditions of this study (Fig. 4C) and others (Holtmaat et al., 2005). However, there is no evidence of which previously persistent spines are eliminated or survive during sensory/activity changes and how the surviving ones behave. Our study show that preexisting stable spines with small head size were more eliminated following PSL than large ones, indicating that neuropathic injury-specific elimination of stable spines is size dependent. The surviving (AP) spines, interestingly, displayed LTP-like enlargement over weeks, suggesting that relatively large preexisting spines are too stable to be eliminated, but their synaptic efficacy is potentiated during neuropathic pain. It should be noted that even very large AP spines showed long-lasting enlargement, to the same proportion as small ones (Fig. $4 C-E$ ), which is somewhat different from in vitro results showing that small spines are susceptible, but large spines are resistant, to activity-dependent enlargement (Yasumatsu et al., 2008).

Even though large-scale reorganization of dendritic and axonal arbors of pyramidal cells in the intact adult cortex within weeks is rare under our experimental conditions (data not shown) and others (Holtmaat and Svoboda, 2009), a minor fraction of persistent synapses added or subtracted by neuropathic injury (Figs. 2-4) and novel experiences can sufficiently store specific long-term information (Bhatt et al., 2009). In addition, immediate local nerve blockade completely prevented the increase in the $\mathrm{S} 1$ spine turnover (Fig. 2E,F) and size (Fig. 4D) as well as tactile allodynia (Figs. $2 E-G, 4 D$ ), showing a direct link between cortical synaptic remodeling and injury-induced early afferent hyperactivity, which is highly associated with neuropathic pain development. Since the same nerve blockade during the late phase (started at PSL $+6 \mathrm{~d}$ ) showed only transient and moderate recovery in mechanical sensitivity $(34.5 \%$ increase at PSL $+9 \mathrm{~d}$, but return to the same level as saline-treated PSL mice from PSL $+12 \mathrm{~d}$ on), our results may suggest the importance of preemptive analgesia, which is based on the "pain memory" concept previously suggested at the spinal cord level (Melzack et al., 2001). Thus, the present study probably extends the pain memory hypothesis to single spines at the cortex level. Because several strategies to reduce the degree of reorganization and hyperexcitability in the S1 showed the benefits against neuropathic pain (Seifert and Maihöfner, 2009), the detailed cellular mechanisms as demonstrated here provide a potential therapeutic target in debilitating chronic pain. Recently, gabapentin, a well known drug for neuropathic pain, was shown to block excitatory CNS synaptogenesis, not affect preexisting synapses (Eroglu et al., 2009). Therefore, it would be of great interest to investigate whether direct application of such drugs into the cortex can pre- 
vent or erase the "pain memory" in specific synapses that in turn abolish chronic pain without affecting normal sensations.

\section{References}

Bhatt DH, Zhang S, Gan WB (2009) Dendritic spine dynamics. Annu Rev Physiol 71:261-282.

Cha MH, Kim DS, Cho ZH, Sohn JH, Chung MA, Lee HJ, Nam TS, Lee BH (2009) Modification of cortical excitability in neuropathic rats: a voltagesensitive dye study. Neurosci Lett 464:117-121.

Costigan M, Scholz J, Woolf CJ (2009) Neuropathic pain: a maladaptive response of the nervous system to damage. Annu Rev Neurosci 32:1-32.

DaSilva AF, Becerra L, Pendse G, Chizh B, Tully S, Borsook D (2008) Colocalized structural and functional changes in the cortex of patients with trigeminal neuropathic pain. PLoS One 3:e3396.

Devor M (2006) Response of nerves to injury in relation to neuropathic pain. In: Wall and Melzack's textbook of pain (McMahon SL, Koltzenburg M, eds), pp 905-927. London: Churchill Livingstone.

Eroglu C, Allen NJ, Susman MW, O’Rourke NA, Park CY, Ozkan E, Chakraborty C, Mulinyawe SB, Annis DS, Huberman AD, Green EM, Lawler J, Dolmetsch R, Garcia KC, Smith SJ, Luo ZD, Rosenthal A, Mosher DF, Barres BA (2009) Gabapentin receptor alpha2delta-1 is a neuronal thrombospondin receptor responsible for excitatory CNS synaptogenesis. Cell 139:380-392.

Feng G, Mellor RH, Bernstein M, Keller-Peck C, Nguyen QT, Wallace M, Nerbonne JM, Lichtman JW, Sanes JR (2000) Imaging neuronal subsets in transgenic mice expressing multiple spectral variants of GFP. Neuron 28:41-51.

Florence SL, Taub HB, Kaas JH (1998) Large-scale sprouting of cortical connections after peripheral injury in adult macaque monkeys. Science 282:1117-1121.

Hofer SB, Mrsic-Flogel TD, Bonhoeffer T, Hübener M (2009) Experience leaves a lasting structural trace in cortical circuits. Nature 457:313-317.

Holtmaat A, Svoboda K (2009) Experience-dependent structural synaptic plasticity in the mammalian brain. Nat Rev Neurosci 10:647-658.

Holtmaat AJ, Trachtenberg JT, Wilbrecht L, Shepherd GM, Zhang X, Knott GW, Svoboda K (2005) Transient and persistent dendritic spines in the neocortex in vivo. Neuron 45:279-291.

Holtmaat A, Wilbrecht L, Knott GW, Welker E, Svoboda K (2006) Experience-dependent and cell-type-specific spine growth in the neocortex. Nature 441:979-983.

Ji RR, Kohno T, Moore KA, Woolf CJ (2003) Central sensitization and LTP: do pain and memory share similar mechanisms? Trends Neurosci 26:696-705.

Kakizawa S, Miyazaki T, Yanagihara D, Iino M, Watanabe M, Kano M (2005) Maintenance of presynaptic function by AMPA receptor-mediated excitatory postsynaptic activity in adult brain. Proc Natl Acad Sci U S A 102:19180-19185.
Kasai H, Matsuzaki M, Noguchi J, Yasumatsu N, Nakahara H (2003) Structure-stability-function relationships of dendritic spines. Trends Neurosci 26:360-368.

Kawasaki Y, Xu ZZ, Wang X, Park JY, Zhuang ZY, Tan PH, Gao YJ, Roy K, Corfas G, Lo EH, Ji RR (2008) Distinct roles of matrix metalloproteases in the early- and late-phase development of neuropathic pain. Nat Med 14:331-336.

Knott GW, Holtmaat A, Wilbrecht L, Welker E, Svoboda K (2006) Spine growth precedes synapse formation in the adult neocortex in vivo. Nat Neurosci 9:1117-1124.

Malmberg AB, Basbaum AI (1998) Partial sciatic nerve injury in the mouse as a model of neuropathic pain: behavioral and neuroanatomical correlates. Pain 76:215-222.

Matsuzaki M, Honkura N, Ellis-Davies GC, Kasai H (2004) Structural basis of long-term potentiation in single dendritic spines. Nature 429:761-766.

Melzack R, Coderre TJ, Katz J, Vaccarino AL (2001) Central neuroplasticity and pathological pain. Ann NY Acad Sci 933:157-174.

Murayama M, Pérez-Garci E, Nevian T, Bock T, Senn W, Larkum ME (2009) Dendritic encoding of sensory stimuli controlled by deep cortical interneurons. Nature 457:1137-1141.

Peyron R, Schneider F, Faillenot I, Convers P, Barral FG, Garcia-Larrea L, Laurent B (2004) An fMRI study of cortical representation of mechanical allodynia in patients with neuropathic pain. Neurology 63:1838-1846.

Pozo K, Goda Y (2010) Unraveling mechanisms of homeostatic synaptic plasticity. Neuron 66:337-351.

Seifert F, Maihöfner C (2009) Central mechanisms of experimental and chronic neuropathic pain: findings from functional imaging studies. Cell Mol Life Sci 66:375-390.

Takatsuru Y, Fukumoto D, Yoshitomo M, Nemoto T, Tsukada H, Nabekura J (2009) Neuronal circuit remodeling in the contralateral cortical hemisphere during functional recovery from cerebral infarction. J Neurosci 29:10081-10086.

Wake H, Moorhouse AJ, Jinno S, Kohsaka S, Nabekura J (2009) Resting microglia directly monitor the functional state of synapses in vivo and determine the fate of ischemic terminals. J Neurosci 29:3974-3980.

Xie W, Strong JA, Meij JT, Zhang JM, Yu L (2005) Neuropathic pain: early spontaneous afferent activity is the trigger. Pain 116:243-256.

Xu HT, Pan F, Yang G, Gan WB (2007) Choice of cranial window type for in vivo imaging affects dendritic spine turnover in the cortex. Nat Neurosci 10:549-551.

Yang G, Pan F, Gan WB (2009) Stably maintained dendritic spines are associated with lifelong memories. Nature 462:920-924.

Yasumatsu N, Matsuzaki M, Miyazaki T, Noguchi J, Kasai H (2008) Principles of long-term dynamics of dendritic spines. J Neurosci 28:13592-13608.

Zhuo M (2008) Cortical excitation and chronic pain. Trends Neurosci 31: 199-207. 